\title{
Software development for computer-assisted instruction in experimental psychology
}

\author{
JAMES E. SPIVEY \\ University of Kentucky, Lexington, Kentucky 40506
}

\begin{abstract}
The introduction of CAI modules was pursued as a means of strengthening an experimental psychology course. After securing 12 Apple II-Plus microcomputers and a Winchester harddisk system, we turned to the search for and development of relevant software. Our efforts were initially frustrated due to several inadequacies in extant software that are discussed herein. A surprising source of software turned out to be the commercial market; two well known commercial programs have been modified for educational use, and one of these programs has provided a pleasant solution to student computerphobia. These two old modules are discussed, along with others that are either borrowed or new. Although total computerization has been avoided for reasons discussed herein, extensive evaluation has indicated that the modules have greatly facilitated the realization of course goals and have especially minimized the three complaints (irrelevant, boring, too difficult) most often heard in traditional research methods courses. In addition, our Attitude Toward Computers Scale indicates that students develop a more positive attitude toward computers as a result of their usage in the course. This positive attitude is seen as most adaptive in a society within which computers will play an increasingly dominant role.
\end{abstract}

In the summer of 1980, the Department of Psychology at the University of Kentucky began the revision of its undergraduate program with a total of one-half million dollars provided by the National Science Foundation (NSF) through a CAUSE (comprehensive assistance to undergraduate science education) grant and matching funds from the university. Although monies are being used for several innovative approaches to curriculum reform, the centerpiece in the master plan is a computerassisted instruction (CAI) laboratory containing 12 Apple II-Plus microcomputers and a Winchester harddisk system (see Lowe, 1983). The two courses to which CAI was viewed as most appropriate were statistics and

This project is supported by National Science Foundation Grant SER-8005325 and matching funds from the University of Kentucky. Ronald C. Dillehay was department chairperson and was primarily responsible for securing the grant; he continues as grant director. Richard G. Davis provided expert counsel on hardware acquisition and configuration and continues to serve in an advisory capacity. The CAI laboratory is supervised by David C. Lowe, and the computer programs were written by David C. Lowe and John A. Walker. External evaluators are James R. Sanders and Daniel L. Stufflebeam; the internal evaluation team, directed by Timothy A. Smith, has consisted of Dennis J. Johnson, Craig J. Petrun, Glen P. Rogers, and Isabella M. Zembrodt. Special appreciation is due Arthur J. Nonneman, who not only served as acting director during the 1 st year of the grant while R. C. Dillehay was on sabbatical but also made numerous substantive contributions to several of the modules. During the planning and development of the present version of the course, I have profited greatly from the advice of Susan M. Belmore, Pamela Jackson-Smith, and Robin Robinson. Sole responsibility for statements contained in this paper lies with the author. Requests for reprints or additional information should be addressed to James E. Spivey, Department of Psychology, Kastle Hall, University of Kentucky, Lexington, Kentucky 40506. experimental psychology. After having taught the experimental psychology course for a number of years, I believed that the introduction of CAI not only could aid in better achieving the overall course goals but, even more important, could minimize three major complaints typically lodged by students in research methods courses.

The course goals include: (1) a working knowledge of the scientific method, (2) an appreciation for the application of (relevance of) the scientific method to the subject matter of modern psychology, (3) an ability to critically evaluate published research, (4) an ability to form testable hypotheses and to design and conduct appropriate experiments as tests of these hypotheses, and (5) an ability to write APA-style research manuscripts with objectivity and clarity.

The three major complaints of students are that research-oriented courses are: (1) not "relevant," (2) boring, with an emphasis on time-consuming busy work such as collecting and handling data, and (3) too difficult.

As expected, the use of CAI not only has allowed a greater realization of course goals but also has precluded the aforementioned complaints. The course, which was well rated in spite of student grumblings, is even more popular.

It is important to point out that the introduction of the CAI approach brought with it two new problems. The first of these is computerphobia. The second is a complex array of artifacts that for a while rendered the most successful traditional experiment useless as a computer simulation in spite of a full year's work on several versions of the module (Spivey \& Jackson-Smith, 1983). 


\section{THE SEARCH}

Our major efforts on this project have been aimed at the search for and/or development of software. It had been assumed, in line with the assurances of consultants, that educational software would be available through Conduit and other sources. A review of available software, much of it after translation into Apple BASIC, led us to conclude that educational software, with few exceptions, had three major weaknesses. First, and most problematic for the experimental psychology course, most modules were only simple demonstrations with no true (manipulable) independent variables. Second, many modules were boring. Third, program documentation of most modules was either nonexistent or incomplete; consequently, it was necessary to abandon several promising programs. Justifying the investment of one's time at this point became a major exercise in dissonance reduction.

In fairness to the developers of these available modules, it was concluded that the faults were primarily due to the date of module creation. Considering the very rapid developments in the computer field, one has to avoid criticism of the true pioneers. In the microcomputer field, the term "pioneer" applies to those involved in module development only 3.5 years prior to our entry into the field.

To guide the search for and development of modules, there were established for each module highly specific goals relevant to the portion of the course during which the module was to be used. Further, it was understood at the outset of the project that the CAI modules would make up only about one-half of the laboratory portion of the course. It was felt that a completely "computerized" laboratory would have an artificial air and would deny students the benefits that accrue from real-life, hands-on, traditional experiments.

Some of the individual modules now need to be discussed to make more specific and to document the general $\mathrm{cr}$. iclusions of the preceding paragraphs.

\section{SOMETHING OLD}

One of our major needs was a module to reduce computerphobia. By the end of the first semester, it had become apparent that computerphobia was contributing to the drop rate early in the semester. Also, student performance on modules suffered from a lack of keyboard familiarity in spite of a verbal orientation and guided student practice with the keyboard. The excessively slow and inconsistent responses of students were most harmful to those modules for which response speed was the dependent variable. One consultant even recommended the purchase of a typing tutor modulenot an exciting prospect.

We cast about for a more suitable module and decided that the Apple game "Animals" was ideal for our pur- poses. The game has been revised and has been retitled "Hypothesis Testing." The program has two animals, a moose and a frog, stored in its repertoire, and it begins by asking the user a question that allows for a possible distinction between the two animals. If the user has a third animal in mind, the program asks him/her to type in a question that will distinguish that animal from the other land- or water-based animal of the original two. After a while, the user has generated a succession of questions that provide stepwise access to the endpoints of a large branching matrix.

Students are asked to participate in pairs to reduce anxiety and to speed learning, and performance on this module is not graded. Each member of a pair must play the game for $25 \mathrm{~min}$. After $50 \mathrm{~min}$, students trade machines and try to find all of the animals stored in another machine's file. This simple, well known program accomplishes as much or more than any other module in our library. Its primary achievement is that it desensitizes the students to the Apple, and computerphobia for them is a thing of the past. Second, the great amount of typing involved in the use of the program familiarizes the students with the keyboard and increases their response speed and consistency to an acceptable level. The third benefit is substantive; the branching nature of the program, with the user asking a series of questions (testing related hypotheses) and being given a "yes" or "no" answer, is akin to scientific research. The students do not lose this point in the "game" atmosphere. Finally, the students begin the course on an optimistic note, and the criticisms of "boring" and "too difficult" begin to fade.

We next wanted a module that would indicate the relevance of the scientific method. For this purpose, another well known program, "Lemonade," was selected and modified. This program allows the user to manipulate a number of independent variables within several different boundary conditions in an effort to make money (the major dependent variable). This program, like the previous one, is deceptively simple in appearance, but it accomplishes a great deal. First, students enjoy it very much and want to remain after class as they do with hypothesis testing. Second, and most important, the program emphasizes the relevance of the scientific method. That is, a successful person in science or business is concerned mainly with cause-effect relationships. Through the use of an elaborate four-page handout that accompanies it, the module accomplishes a third purpose in reviewing and illustrating the first five chapters of the course text (Matheson, Bruce, \& Beauchamp, 1978).

\section{SOMETHING BORROWED}

Another needed module was one that would allow the students to test their skills at designing a real experiment. We felt that a good computer simulation could 
not be beaten for that purpose. Students at this point in the course have already run two traditional paper-andpencil experiments in the laboratory. One is a cognitive dissonance experiment for which they are given a poor write-up for correction in the laboratory; the second is a sexism experiment that students analyze and write up in APA style. In spite of this, students are convinced that they cannot create an experiment.

In the search for a good simulation module, we fortunately came across David Gibson's "Pavlov." After revision and elaboration, this module accomplishes very nicely the purposes for which it was sought. The module allows students to set a value for a number of independent variables (e.g., level of training, deprivation, sex of subjects) and generates appropriate responses (number of drops of saliva) for that particular subject for each trial of acquisition and extinction. We have found that it is best not to give students too much guidance but, rather, to let them find whether they can work through the module alone and come up with a good design. After $15 \mathrm{~min}$, students are asked to come to an adjoining classroom where they illustrate their designs on a chalkboard. Within about $15 \mathrm{~min}$, either through self-correction or peer guidarice (the teaching assistant says as little as possible), everyone has a good design and returns to the laboratory to complete the running of their "subjects." Students are not asked to write up this experiment, but to organize the data on large data sheets with the appropriate location of the cells of the repeated-measures design. This not only teaches them how to organize large amounts of data but also tests and reinforces their conceptualizations of designs. Students then statistically evaluate and graph their results.

This module accomplishes several objectives. The first is the aforementioned experience with handling repeated-measures data. Second, it reinforces basic information about experimental design and control. Third, it greatly bolsters student confidence in their ability to perform as scientists, which they find they enjoy. This third factor, along with the historical importance of Pavlov's original work, generates high student interest in the module.

\section{SOMETHING NEW}

If Hypothesis Testing and Lemonade are old, and Pavlov is borrowed, then the last two modules currently employed are new.

The fourth module, "Shapes," is discussed elsewhere (Spivey \& Jackson-Smith, 1983). Here, it is enough to say that our experience with that module illustrates that CAI or the computerization of traditional laboratory modules is not always for the best.

The final module is titled "Statement Verification" and consists of a number of statements in each of four forms: true, false, negated true, and negated false. The dependent variable is the percent correct answers and the average response speed for each of the four types of questions. Each subject, through the use of the Win. chester hard-disk system, can trace his/her responses and test a number of hypotheses. These hypotheses deal not only with treatment or summary data, such as percent correct or overall response speeds to the four types of items, but also with processes such as interference result ing from the presentation of a negated false statement after the same statement in its true form.

The primary goal of this module is to provide experience with single-subject designs. Students become aware of the richness of an intensive study of the responses of a single individual (themselves) and the possible limitations on generalizations. A second goal of the module is the comparison of treatment with process data and the recognition of the very different roles the two types of information can play.

\section{TOTAL COMPUTERIZATION AVOIDED}

It was mentioned earlier that the laboratory has not been totally converted into a computer-generated tutorial. No matter how interesting the CAI modules, all but the most extreme computerphiles prefer a variety of educational modalities. Further, students need experience with some of the traditional materials and methods employed by psychologists. A traditional animal experiment is used as the final course wrap-up. Students must maintain and run rats in a 2 -week experiment, after which they are asked to provide their second major laboratory paper written in APA style (the first paper was based on the sexism experiment early in the semester).

\section{EVALUATION}

Evaluation has played a large role in the CAUSE project in response both to the desires of project personnel and to the requirements of NSF. The selection and development of evaluation instruments, as with the CAI modules, have been tied to course goals.

A large part of our evaluation is, of course, based on student performance on the modules (each of which is accompanied by a written test of content and an evaluation of the module), on course examinations, and on the two major APA-style papers. These three sources of information indicate that the course goals are being realized. Not only do the students perform well on the modules, but also they perform on course examinations at a higher level than did previous classes. Even more interesting is the finding that student improvement on the two APA-style papers from the sexism (traditional) module early in the course to the rat (traditional) experiment late in the semester is as great as the improvement found with four to six intervening write-ups required in the previous version of this course. The conclusion we have reached is that the CAI modules 
now are teaching what previously was taught through writing laboratory reports with less effort and with far less student resistance.

Written student evaluations taken at the end of the semester and not viewed by instructors until after grades are turned in reveal a marked decrease in student hostility toward the course. Students do not find the course to be boring or too difficult; further, students do not report the course to be irrelevant. Students, in fact, rate the course very highly. These evaluations, as well as conversations with students, reveal that students have a great appreciation for and an understanding of the place of the scientific method and of computers in modern life.

The primary objective evaluation instruments are three attitude scales given to students on the 1st day of class and one experimental psychology (achievement) test given on the 2nd class day. Each of these four pretests is again administered as a posttest at the end of the course. The experimental psychology test difference scores indicate not only that students learn a great deal, but also that those items relevant to CAI modules are those that show the greatest positive change scores. The Attitude Toward Computers Scale, developed locally, indicates an increasingly positive attitude toward computers as a result of the course. The Attitude Toward Science and Attitude Toward Psychology scales originated at the University of Michigan (Carrier, 1966) and were adapted for our use. Unfortunately, the differences in scores indicate only a small positive change overall; however, a larger positive change was found for those items on the Attitude Toward Science Scale that have to do with the relevance of science or the scientific method to the immediate, practical problems of society. Fortunately, these items are those that are viewed as most important.

As experimental psychologists, we realize that what is most needed for the evaluation of the efficacy of CAl are controlled experiments. Belmore's (1983) recent attempt in this direction seems to indicate a lack of the expected superiority of a CAI version of her module over a traditional version of the same module. More research such as this needs to be done. However, no one would argue that all laboratory modules are best done on a computer, but rather, that some certainly are.

The most parsimonious interpretation of all of the evaluation data would be that the introduction of CAI has increased student interest and learning. How much of this increase is due to CAI itself and how much is due to the increased variety of teaching modes is not possible at present to determine.

\section{SUMMARY}

In spite of early disappointments in the applicability of existing software to our needs and setbacks resulting from unanticipated human-factors problems, the benefits of the introduction of CAI into the experimental psychology course are not yet fully realized. It is concluded at this point that through the use of microcomputers, students' resentment of and resistance to research methods courses can be replaced with the realization of a rich and highly enjoyable educational experience.

\section{REFERENCES}

Belmone, S. M. RELEASE FROM PI: Comparison of traditional and computer modules in an experimental psychology laboratory. Behavior Research Methods \& Instrumentation, 1983, 15, 191-194.

Carrier, N. A. Evaluating the introductory psychology course. Reading, Mass: Addison-Wesley, 1966.

LowE, D. C. A Winchester hard-disk integrated computer-assisted instructional laboratory: Hardware and data management considerations. Behavior Research Methods \& Instrumentation, 1983, 15, 181-182.

Matheson, D. W., Bruce, R. L., \& Beauchamp, K. L. Experimental psychology: Research design and analysis (3rd ed.). New York: Holt, Rinehart, \& Winston, 1978.

SpIveY, J. E., \& Jackson-Smith, P. Computer simulation: A case of conflict. Behavior Research Methods \& Instrumentation, $1983,15,187-190$. 